\title{
2-Chlorofatty Aldehyde Elicits Endothelial Cell Activation
}

\author{
Jane McHowat ${ }^{1,2}$, Shubha Shakya ${ }^{2,3}$ and David A. Ford ${ }^{2,3 *}$ \\ ${ }^{1}$ Department of Pathology, Saint Louis University School of Medicine, St. Louis, MO, United States, ${ }^{2}$ Center \\ for Cardiovascular Research, Saint Louis University School of Medicine, St. Louis, MO, United States, ${ }^{3}$ Edward A. Doisy \\ Department of Biochemistry and Molecular Biology, Saint Louis University School of Medicine, St. Louis, MO, United States
}

\section{OPEN ACCESS}

Edited by:

David H. Wasserman, Vanderbilt University, United States

Reviewed by:

Nathan C. Winn, Vanderbilt University, United States Ian Williams,

Stanford University School of Medicine, United States

*Correspondence:

David A. Ford

david.ford@health.s/u.edu

Specialty section:

This article was submitted to Lipid and Fatty Acid Research, a section of the journal Frontiers in Physiology

Received: 30 October 2019 Accepted: 16 April 2020 Published: 08 May 2020

Citation:

McHowat J, Shakya S and Ford DA (2020) 2-Chlorofatty Aldehyde Elicits Endothelial Cell Activation. Front. Physiol. 11:460. doi: 10.3389/fphys.2020.00460
Endothelial activation and dysfunction are hallmarks of inflammation. Neutrophilvascular endothelium interactions have significant effects on vascular wall physiology and pathology. Myeloperoxidase (MPO)-derived products released from activated neutrophils can mediate the inflammatory response and contribute to endothelial dysfunction. 2-Chlorofatty aldehyde (2-CIFALD) is the direct oxidation product of MPO-derived hypochlorous acid $(\mathrm{HOCl})$ targeting plasmalogen phospholipids. The role of 2-CIFALD in endothelial dysfunction is poorly understood and may be dependent on the vascular bed. This study compared the role of 2-CIFALD in eliciting endothelial dysfunction in human coronary artery endothelial cells (HCAEC), human lung microvascular endothelial cells (HLMVEC), and human kidney endothelial cells (HKEC). Profound increases in selectin surface expression as well as ICAM-1 and VCAM-1 surface expression were observed in HCAEC and HLMVEC. The surface expression of these adherence molecules resulted in robust adherence of neutrophils and platelets to 2-CIFALD treated endothelial cells. In contrast to HCAEC and HLMVEC, 2-CIFALDtreated HKEC had substantially reduced adherence molecule surface expression with no resulting increase in platelet adherence. 2-CIFALD-treated HKEC did have an increase in neutrophil adherence. All three endothelial cell lines treated with 2-CIFALD displayed a time-dependent loss of barrier function. Further studies revealed 2-CIHDyA localizes to ER and Golgi when using a synthetic alkyne analog of 2-CIFALD in HCAEC and HLMVEC. These findings indicate 2-CIFALDs promote endothelial cell dysfunction with disparate degrees of responsiveness depending on the vascular bed of origin.

Keywords: endothelial cells, fatty acids, inflammation, lipid mediators, plasmalogens, vascular biology

\section{INTRODUCTION}

Changes at the blood-endothelial interface can elicit profound changes in organ physiology. Leukocytes can initiate an inflammatory response in the vascular wall resulting in the release of inflammatory mediators and oxidants (Szekanecz and Koch, 2004; Langer and Chavakis, 2009), which may lead to dysfunction at the blood-endothelium interface. Endothelial dysfunction is an early event in the pathophysiological sequelae of diseases including atherosclerosis,

Abbreviations: 2-ClFALD, 2-chlorofatty aldehyde; 2-ClHDA, 2-chlorohexadecanal; 2-ClHDyA, 2-chlorohexadec-15-ynal; HCAEC, human coronary artery endothelial cell; HDA, hexadecanal; HDyA, hexadec-15-ynal; MPO, myeloperoxidase; PFB$\mathrm{Br}$, pentafluorobenzyl bromide; VWF, von Willebrand factor. 
ischemia/reperfusion injury in the heart, and in sepsis multiorgan failure (Ijzerman et al., 2003; Crea et al., 2014; De Backer et al., 2014). Once activated the endothelium releases many mediators that can contribute to leukocyte adherence, coagulation, and changes in barrier function (Ait-Oufella et al., 2010). Through this escalating inflammation, leukocyteproduced reactive oxygen species and released proteolytic enzymes propagate tissue damage and are involved in organ failure (Rossaint and Zarbock, 2015; Santos et al., 2016).

Myeloperoxidase is the most abundant protein in the neutrophil and is a key antimicrobial enzyme released by activated neutrophils (Ysebaert et al., 2000; Klebanoff et al., 2013). During neutrophil activation sequential NADPH oxidase production of hydrogen peroxide and MPO conversion of hydrogen peroxide and chloride anion lead to the production of $\mathrm{HOCl}$. $\mathrm{HOCl}$ is a potent antimicrobial agent capable of oxidizing proteins, free amino acids, carbohydrates, DNA, and lipids (Harrison and Schultz, 1976; Hazen et al., 1996; Pattison and Davies, 2006; Pattison et al., 2009). Plasmalogens, a major phospholipid in many human organs, are targeted by $\mathrm{HOCl}$ leading to the production of 2-ClFALDs (Dorman et al., 1976; Chilton and Connell, 1988; Ford and Gross, 1989; Murphy et al., 1992; Hazen et al., 1993; Portilla and Creer, 1995; McHowat et al., 1997; Albert et al., 2001; Hsu et al., 2003). 2-ClFALDs can enter the fatty acid-fatty alcohol cycle as an intermediate leading to their oxidation to 2-chlorofatty acids (2-ClFAs) (Rizzo et al., 1987; Wildsmith et al., 2006; Anbukumar et al., 2010; Brahmbhatt et al., 2010). During inflammation, 2-ClFALDs and 2-ClFAs are produced at the site of neutrophil infiltration and likely alter nearby cell function (Ford, 2010).

2-Chlorofatty aldehyde and 2-ClFA have been linked to inflammatory diseases, including endotoxemia and atherosclerosis (Thukkani et al., 2003, 2005; Brahmbhatt et al., 2010; Ford et al., 2016). Activated neutrophils and monocytes isolated from human blood have elevated levels of 2-ClFALD, approaching 20-90 $\mu \mathrm{M}$ (compared to undetectable levels in inactivated neutrophils and monocytes), which represents an approximation of the maximal concentrations at the site of production near the leukocyte-endothelial interface (Thukkani et al., 2002; Anbukumar et al., 2010). Other studies have also shown neutrophil-derived $\mathrm{HOCl}$ can target endothelial plasmalogens resulting in endothelial 2-ClFALD production (Thukkani et al., 2002). Chlorinated lipids have been shown to elicit recruitment of macrophages to the lung, disrupt the blood-brain barrier, and induce apoptosis (Wang et al., 2014; Ford et al., 2016; Nusshold et al., 2016). Recently 2-ClFALD was shown to increase leukocyte rolling and adhesion in the mesenteric microcirculation (Yu et al., 2018). Despite these insights into the biological role of chlorinated lipids the role of 2-ClFALD and 2-CIFA elicited endothelial dysfunction remain to be further characterized. In particular, the demonstration that 2-ClFA associates with ARDS in humans with sepsis (Meyer et al., 2017) suggests understanding the role of 2-CIFALD, the precursor of 2-ClFA, on tissue specific endothelium is needed. Accordingly, the present studies were designed to elucidate mechanisms responsible for 2-CIFALD mediated endothelial activation and distinguish mechanisms in endothelial cells from several vascular beds. Furthermore, a novel click chemistry approach was used to determine the subcellular localization of 2-CIFALD in vascular endothelial cells.

\section{MATERIALS AND METHODS}

\section{Materials}

Cell culture supplies were purchased from Sigma-Aldrich (Vienna, Austria), Lonza (Basel, Switzerland), or Cell Applications Inc. (San Diego, CA, United States). Click-It Cell Reaction Buffer Kit was purchased from Thermo Fisher Scientific (Waltham, MA, United States; catalog no. C10269). Rabbit polyclonal anti-calnexin (catalog no. ab22595), rabbit monoclonal anti- voltage-dependent anion-selective channel 1/porin antibody (anti-VDAC1; catalog no. ab154856), mouse polyclonal anti-golgi matrix protein 130 (anti-GM130; catalog no. ab169276), mouse monoclonal anti-Von Willebrand Factor (anti-VWF; catalog no. ab20435), goat anti-rabbit IgG H\&L (Alexa Fluor $^{\circledR}$ 488) (catalog no: 150077), and goat anti-mouse IgG H\&L (Alexa Fluor ${ }^{\circledR}$ 488) (catalog no. ab150113) antibodies were purchased from Abcam (Cambridge, United Kingdom). Antibodies to adhesion molecules (mouse monoclonal anti-Pselectin, sc-18834; mouse monoclonal anti-E-selectin, sc-5262; mouse monoclonal anti-intercellular adhesion molecule-1, ICAM-1, sc-53336; mouse monoclonal anti-vascular cell adhesion molecule-1, VCAM-1, sc-13160) were purchased from Santa Cruz Biotechnology (Dallas, TX, United States). All other chemicals were purchased from Sigma-Aldrich or Thermo Fisher Scientific.

\section{Synthesis of 2-Chlorohexadec-15-ynal (2-CIHDyA)}

The alkyne analog of 2-ClHDA (2-ClHDyA), was synthesized according to protocols described previously (Halland et al., 2004; Nusshold et al., 2016). Sequentially, (1) hexadec-7ynol (Alfa Aesar, cat. B22113) was converted to hexadec15-ynol using sodium hydride (Sigma-Aldrich, cat. 452912) and diaminopropane (Sigma-Aldrich, cat. D23602) (Nusshold et al., 2016), hexadec-15-ynol was oxidized to HDyA in a solution of 2-iodoxybenzoic acid (Sigma-Aldrich, cat. 661384) in DMSO (Sigma-Aldrich, cat. D2650) (Nusshold et al., 2016), and HDyA was chlorinated using $N$-chlorosuccinimide (SigmaAldrich, cat. 109681) and proline (Sigma-Aldrich, cat. P0380) for $16 \mathrm{~h}$ (Halland et al., 2004). Products were purified by flash chromatography (30 g silica gel, high purity grade, pore size $60 \AA$, Sigma-Aldrich, cat. 227196) (Hartman et al., 2018). 2-ClHDyA was subsequently quantitated by GC-FID following conversion to its dimethyl acetal derivative using heptadecanoic acid and its methyl ester derivative as internal standard (Gross, 1985; Albert et al., 2001).

\section{Endothelial Cell Culture}

Human coronary artery endothelial cells (HCAEC, Lonza, cat. CC-2585) and human lung microvascular endothelial cells (HLMVEC, Lonza, cat. CC-2527) were grown in EGM-2MV 
medium (Lonza, cat. CC-3202). Human renal glomerular endothelial cells (HKEC, Sciencell, cat. 4000) were grown in endothelial cell medium containing 5\% FBS (Sciencell, cat. 1001). Endothelial cells were used in experiments at passage 4-8. Cells were treated with lipids in DMSO.

\section{Localization of 2-CIHDyA in HCAEC and HLMVEC}

Cells were grown to confluence on sterile coverslips and treated with $10 \mu \mathrm{M} 2$-ClHDyA, or no lipid, for $30 \mathrm{~min}$ or $60 \mathrm{~min}$. Cells were washed with PBS, fixed with formalin, and permeabilized with $0.25 \%$ Triton X-100 for $10 \mathrm{~min}$. In the case of VDAC1, cells were permeabilized with ice cold $100 \%$ methanol for $10 \mathrm{~min}$ at $-20^{\circ} \mathrm{C}$. Cells were subsequently washed with $2 \%(\mathrm{w} / \mathrm{v})$ BSA in PBS and labeled with $5 \mu \mathrm{M}$ azidecarboxytetramethylrhodamine (azide-TAMRA) (Sigma-Aldrich; catalog no. 760757) by using the Click-It Cell Reaction Buffer Kit (Thermo Fisher; catalog no. C10269) following manufacturer's protocols. After click reaction, cells were washed with $2 \%$ BSA in PBS. To identify subcellular localization of 2-ClHDyA, cells were incubated with primary antibodies against VDAC1 (1:1000), GM130 (1:142), calnexin (1:1000), and VWF (1:100) overnight at $4{ }^{\circ} \mathrm{C}$. Cells were washed three times with $\mathrm{PBS}$ for $5 \mathrm{~min}$ and incubated with goat antimouse IgG secondary antibody (1:500) labeled with Alexa 488 or goat anti rabbit IgG secondary antibody (1:500) for $1 \mathrm{~h}$. The coverslips were mounted onto microscope slides with a Vectashield solution containing 4',6-diamidino-2-phenylindole (DAPI; Vector Laboratories; catalog no. H1200).

\section{Confocal Microscopy}

A Leica SP5 confocal microscope (Leica Microsystems, Mannheim, Germany) with a $63 \times 1.4$ oil immersion objective was used to acquire images. Alexa 488 was excited at $488 \mathrm{~nm}$ and detected between 500 and $540 \mathrm{~nm}$. Azide-TAMRA fluorescence was excited at $543 \mathrm{~nm}$ and detected between 570 and $650 \mathrm{~nm}$. DAPI fluorescence was detected between 440 and $470 \mathrm{~nm}$. Alexa 488 and TAMRA fluorescence signals were acquired simultaneously.

\section{Adhesion Molecule Surface Expression}

Human coronary artery endothelial cells, HLMVEC, and HKEC were grown to confluence and treated with either $10 \mu \mathrm{M} 2$ ClHDA or HDA for $30 \mathrm{~min}$ (P-selectin), $1 \mathrm{~h}$ (E-selectin) or $4 \mathrm{~h}$ (ICAM-1 and VCAM-1). Cells were not permeabilized, but were fixed with $1 \%$ paraformaldehyde overnight and cell surface expression of adhesion molecules measured as described previously (Meyer et al., 2017; Hartman et al., 2018).

\section{Platelet and Neutrophil Adherence to Endothelial Cells}

Adherence of platelets to either HCAEC, HLMVEC or HKEC was determined as previously described (Verheul et al., 2000). Platelets were isolated from the whole blood of healthy volunteers as previously described (Beckett et al., 2007), and as authorized by Saint Louis University Institutional Review Board Protocol

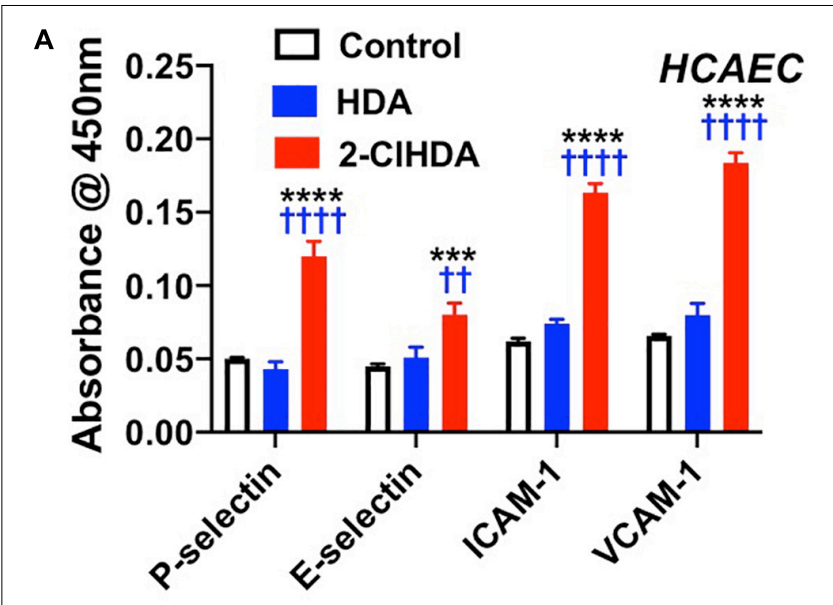

B

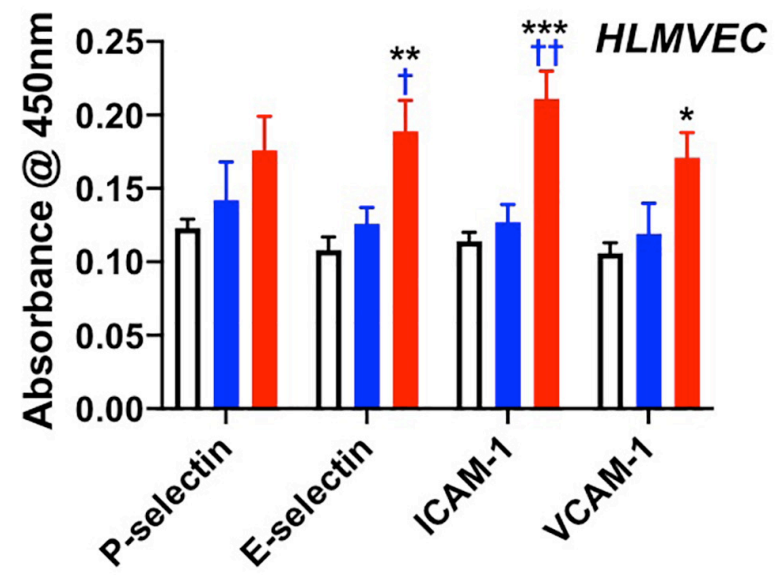

C

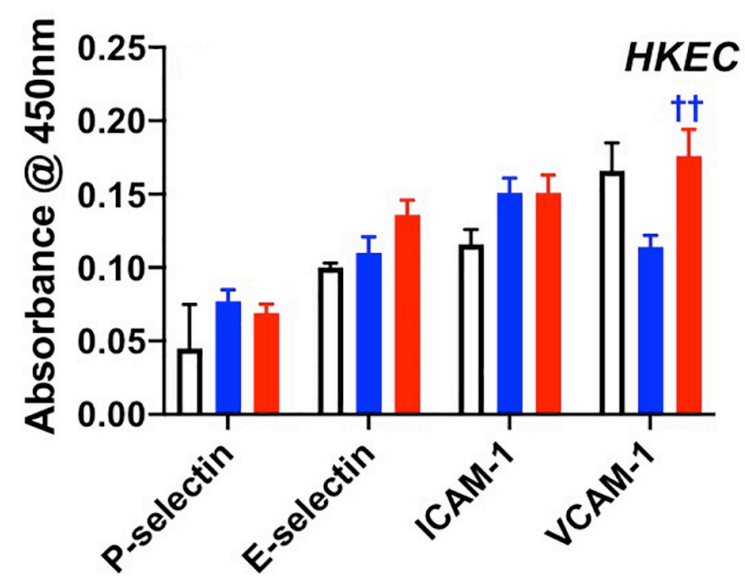

FIGURE 1 | Effects of 2-CIHDA on selectin, ICAM-1 and VCAM-1 surface expression in HCAEC (A), HLMVEC (B), and HKEC (C). Cells were treated with $10 \mu \mathrm{M}$ HDA, $10 \mu \mathrm{M}$ 2-CIHDA or vehicle for 0.5 (P-selectin), $1 \mathrm{~h}$ (E-selectin) or $4 \mathrm{~h}$ (VCAM-1 and ICAM-1). Cells were incubated with primary antibodies and then incubated with HRP-conjugated secondary antibodies. Surface expression of selectins, ICAM-1 and VCAM-1 was measured spectrophotometrically after addition of TMB substrate. Values are normalized to secondary antibody alone. $n=4$ for each treatment, mean \pm SEM. ${ }^{\star} P<0.05,{ }^{\star \star} P<0.01,{ }^{\star \star \star} P<0.001$, and ${ }^{* \star \star \star} P<0.0001$ for comparisons with control treatment. ${ }^{\dagger} P<0.05,{ }^{\dagger \dagger} P<0.01$, and ${ }^{\dagger \dagger \dagger \dagger} P<0.0001$ for comparisons with HDA treatment. 
12369. Platelets were stained with Calcein-AM $(2.5 \mu \mathrm{mol} / \mathrm{L}$; Thermo Fisher, cat. C3100MP) for $15 \mathrm{~min}$ at $37^{\circ} \mathrm{C}$ in the dark. Fluorescence-labeled platelets $\left(50 \times 10^{6}\right.$ cells in $\left.500 \mu \mathrm{L}\right)$ were added to endothelial cells and incubated for $20 \mathrm{~min}$ at $37^{\circ} \mathrm{C}$. Platelet adherence was determined by fluorescence measurement (excitation at $492 \mathrm{~nm}$, emission at $535 \mathrm{~nm}$ ). Neutrophils were prepared from whole blood of healthy volunteers as previously described (Thukkani et al., 2002; Duerr et al., 2015), and as authorized by Saint Louis University Institutional Review Board Protocol 10014. $2 \times 10^{6}$ neutrophils were added to endothelial cells and incubated for $20 \mathrm{~min}$. Neutrophil adherence was measured as MPO activity using 1,9-dimethyl-methylene blue, and absorbance measured at $460 \mathrm{~nm}$ as described previously (Meyer et al., 2017; Hartman et al., 2018).

\section{Endothelial Cell Permeability}

Endothelial cells were grown to confluence on Transwell polycarbonate filters (Corning Inc., Corning, NY, United States) mounted in a chamber insert. Resistance across cells was monitored daily using an EVOM volt-ohmmeter (World Precision Instruments). The lipids, 2-ClHDA or HDA, $(10 \mu \mathrm{M})$ were added to each well then the resistance across each well was monitored up to $24 \mathrm{~h}$.

\section{Cell Viability Assay}

The metabolic activity of HLMVEC was examined using the 3-(4,5-dimethyl-2-thiazolyl)-2,5-diphenyl-2Htetrazolium bromide (MTT) assay as previously described
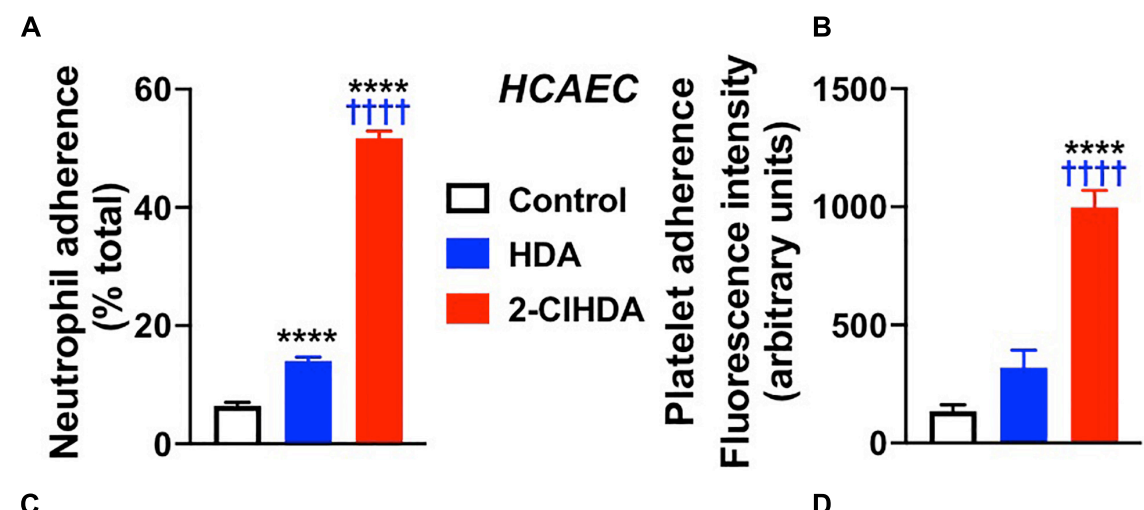

C
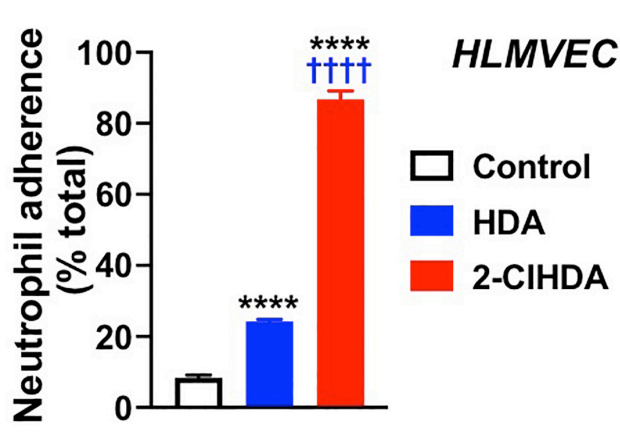

E
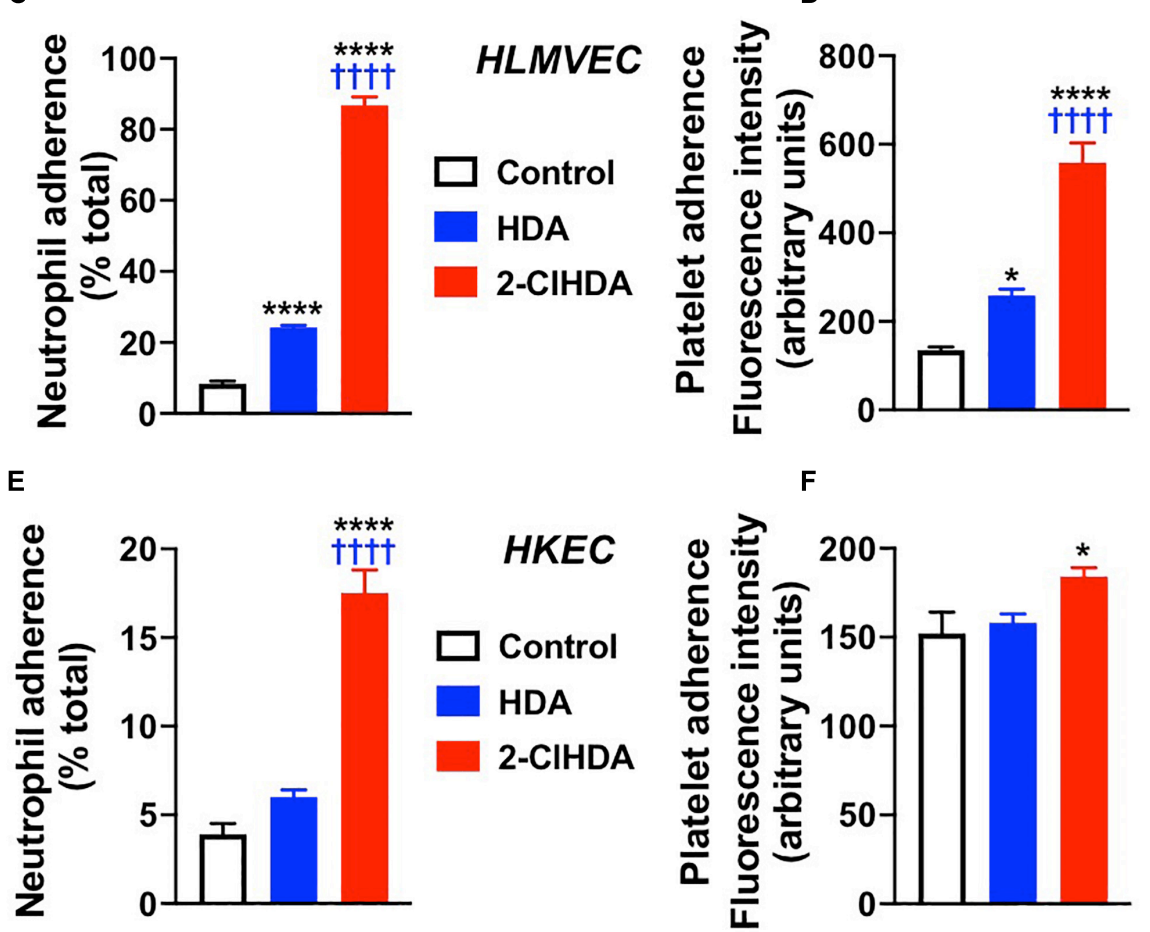

FIGURE 2 | Effects of 2-CIHDA on Neutrophil (A,C,E) and Platelet (B,D,F) Adherence to HCAEC (A,B), HLMVEC (C,D) and HKEC (E,F). Cells were treated with $10 \mu \mathrm{M} \mathrm{HDA}, 10 \mu \mathrm{M}$ 2-CIHDA or vehicle for $4 \mathrm{~h}$. Subsequently endothelial cells were incubated with either freshly isolated human neutrophils or platelets for 20 min to assess adherence. $n=4$ for each treatment, mean \pm SEM. ${ }^{*} P<0.05$, and ${ }^{\star \star \star \star \star} P<0.0001$ for comparisons with control treatment. ${ }^{\dagger+\dagger \dagger} P<0.0001$ for comparisons with HDA treatment. 
(Hartman et al., 2018) to test changes in metabolic activity elicited by either no lipid, 2-ClHDA or 2-ClHDyA $(10 \mu \mathrm{M})$.

\section{Statistical Analyses}

ANOVA with the Tukey's post hoc test was used for comparisons between three groups. All data are presented as mean \pm SEM unless otherwise noted.

\section{RESULTS}

\section{Effect of 2-CIHDA on Surface Expression of Adhesion Molecules as Well as Neutrophil and Platelet Adherence}

Previous studies have shown 2-ClHDA increases leukocyte rolling and adhesion in the mesenteric microcirculation (Yu et al., 2018). To better understand mechanisms responsible for in vivo leukocyte rolling and adhesion we examined the role of 2-ClHDA as a mediator of both adhesion molecule surface expression as well as adhesion of neutrophils and platelets in isolated endothelial cells. Furthermore these studies were performed with human endothelial cells from several vascular beds including HCAEC, HLMVEC and HKEC. Data shown in Figure 1A highlight the responsiveness of HCAEC to 2ClHDA treatment. Significant increases in surface expression of P-selectin, E-selectin, ICAM-1, and VCAM-1 in response to 2ClHDA compared to treatments with either vehicle (control) or HDA. The differential response to 2-ClHDA compared to HDA reveals the importance of the $\alpha$-carbon chlorination of the aldehyde. In comparison to HCAEC, HLMVEC (Figure 1B) had a weaker response to 2-ClHDA treatment, but did show significant increases in E-selectin, ICAM-1, and VCAM-1 when compared to control conditions. Again, HDA did not cause surface expression of these molecules in HLMVEC. In a separate study, cell surface expression of adhesion molecules in response to 2-ClHDA was compared to LPS incubation $(50 \mathrm{ng} / \mathrm{ml})$ in HLMVEC. Similar, but smaller, increases were observed in response to 2-ClHDA when compared to LPS for P-selectin $(0.170 \pm 0.0052$-ClHDA vs. $0.188 \pm 0.005$ LPS $)$, E-selectin $(0.135 \pm 0.0032$-ClHDA vs. $0.258 \pm 0.008$ LPS $)$, ICAM$1(0.189 \pm 0.0062$-ClHDA vs. $0.258 \pm 0.006$ LPS $)$, and VCAM-1 (0.219 \pm 0.0072 -ClHDA vs. $0.253 \pm 0.006$ LPS $)$. Surface expression of the selectins and ICAM-1 did not significantly increase in HKEC in comparison to control conditions (Figure 1C).

Selectin adhesion molecules mediate the adherence of leukocytes to the endothelium (Etzioni, 1996; Peyvandi et al., 2011). Because these molecules are increased following 2ClHDA treatment, the adherence of neutrophils and platelets to endothelium after 2-ClHDA treatment was examined. In concert with elevated surface expression of adhesion molecules, both neutrophil and platelet adhesion to HCAEC and HLMVEC in response to 2-ClHDA was robustly increased in both HCAEC and HLMVEC in comparison to treatments with vehicle as well as in response to HDA (Figures 2A-D).
Interestingly although 2-ClHDA mediated neutrophil adherence to HCAEC and HLMVEC was $\sim 4$-fold greater than that elicited by HDA, significant adherence of neutrophils in response to HDA was detected in comparison to vehicle treatments. Although both platelet and neutrophil adherence to HKEC was significantly increased in response to 2ClHDA treatments (Figures 2E,F), the response in HKEC was weaker compared to that observed in HCAEC and HLMVEC. Additionally HDA did not elicit neutrophil or platelet adhesion to HKEC.

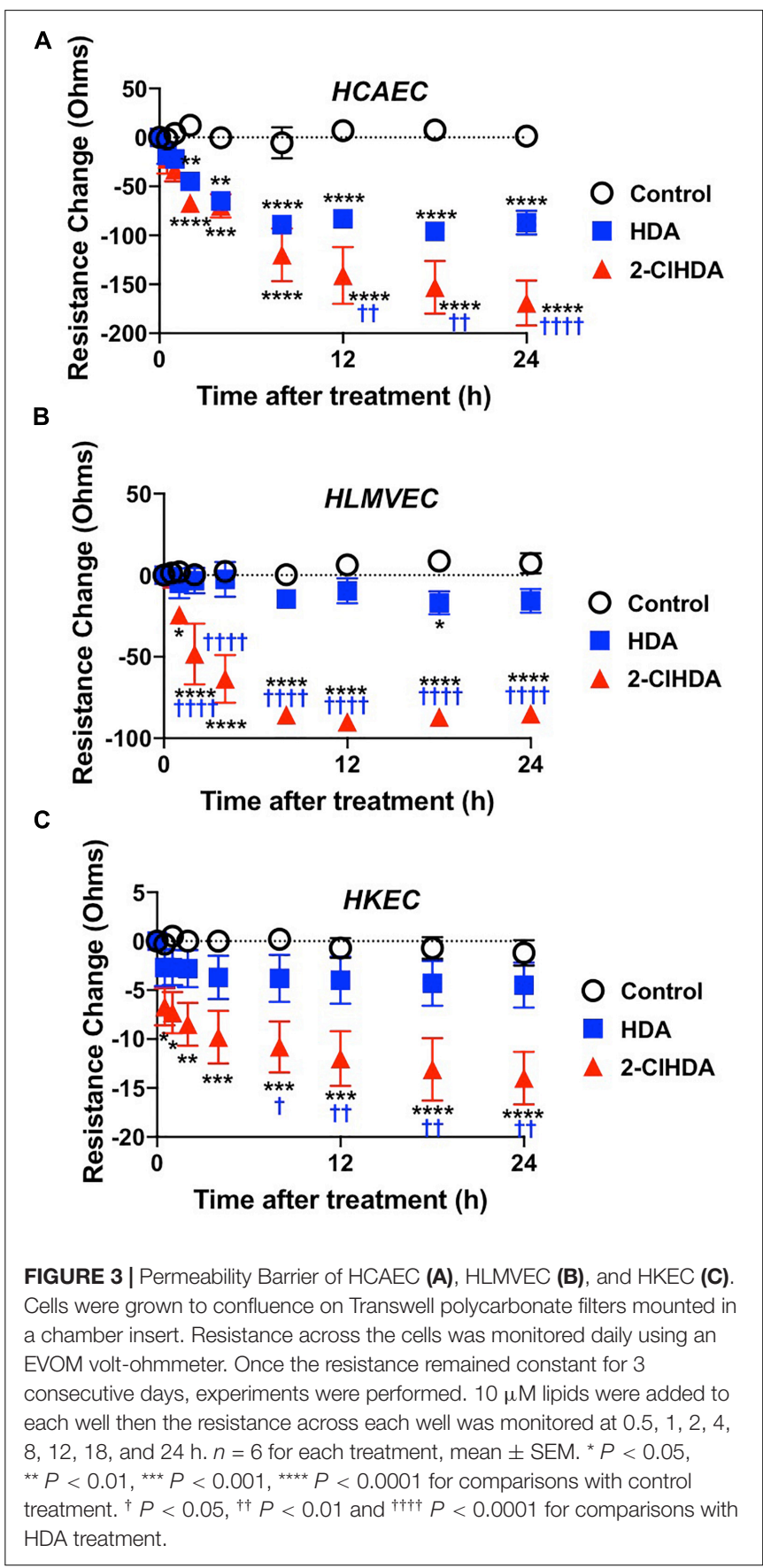




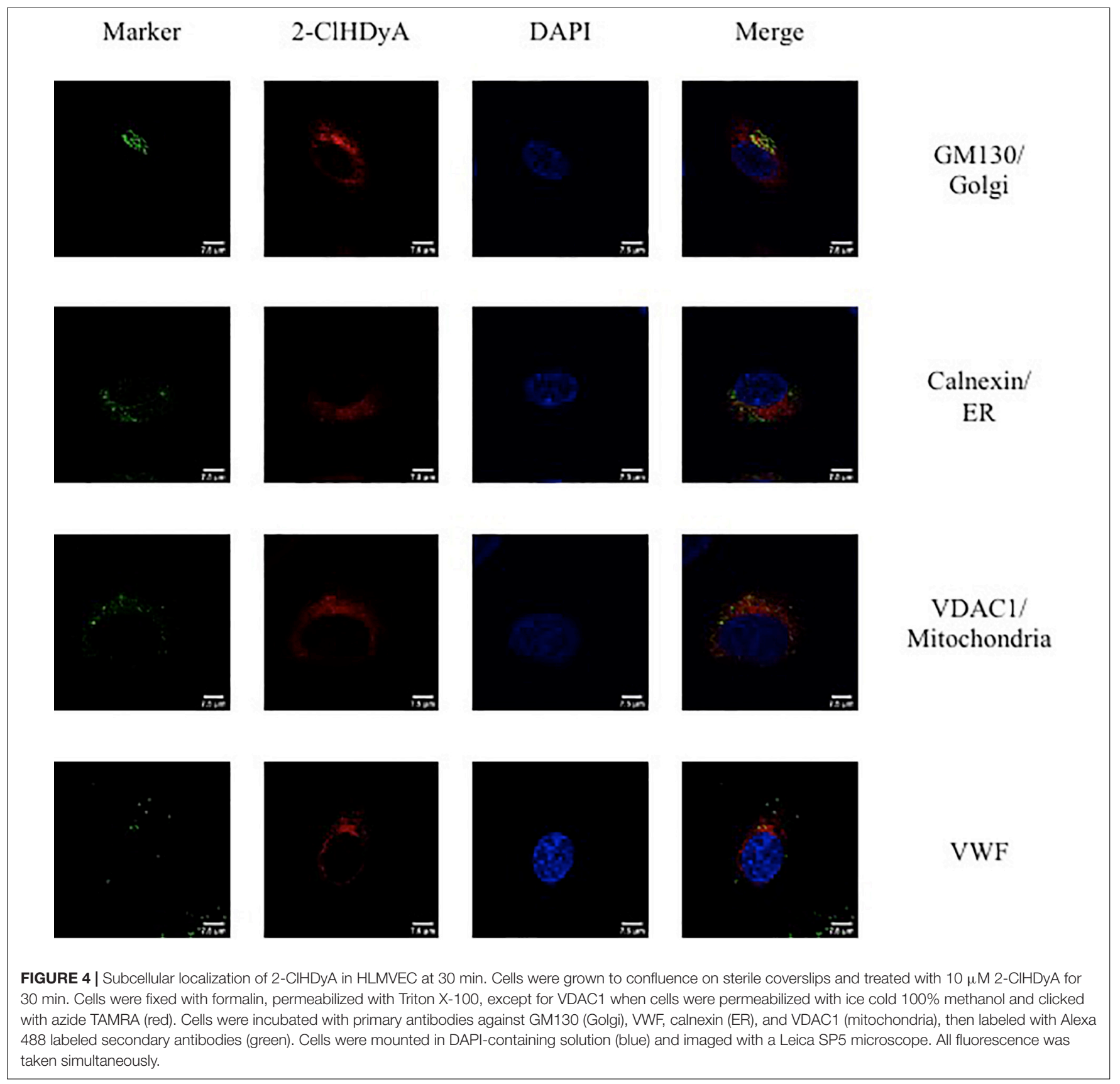

\section{Endothelial Cell Monolayer Permeability in Response to 2-CIHDA}

Similar to conditions comparing adhesion molecule surface expression and neutrophil adhesion to endothelial cells, the impact of 2-ClHDA on endothelial permeability barrier function was determined. HCAEC, HLMVEC, and HKEC all significantly lost barrier function, measured as changes in electrical resistance, in response to 2-ClHDA treatments in comparison to vehicle control treatment (Figures 3A-C). Comparing the response of the three endothelial cell lines to 2-ClHDA indicated HCAEC > HLMVEC > HKEC. Additionally, HCAEC was uniquely susceptible to HDA-mediated loss of barrier function albeit this loss was not as great as that elicited by 2 -ClHDA (Figure 3A). Changes in electrical resistance in response to 2ClHDA were similar to those observed when HLMVEC were incubated with LPS ( $50 \mathrm{ng} / \mathrm{ml}$ ) for up to $24 \mathrm{~h}$ (data not shown).

\section{Subcellular Localization of 2-CIHDyA}

Since HLMVEC and HCAEC gave robust responses to 2-CIHDA, additional studies were performed to examine the subcellular distribution of 2-ClHDA in these cells. For these studies the clickchemistry analog of 2-ClHDA, 2-ClHDyA, was used. Following either a $30 \mathrm{~min}$ or $60 \mathrm{~min}$ incubation of 2-ClHDyA with endothelial cells, cells were fixed and then click reactions were 


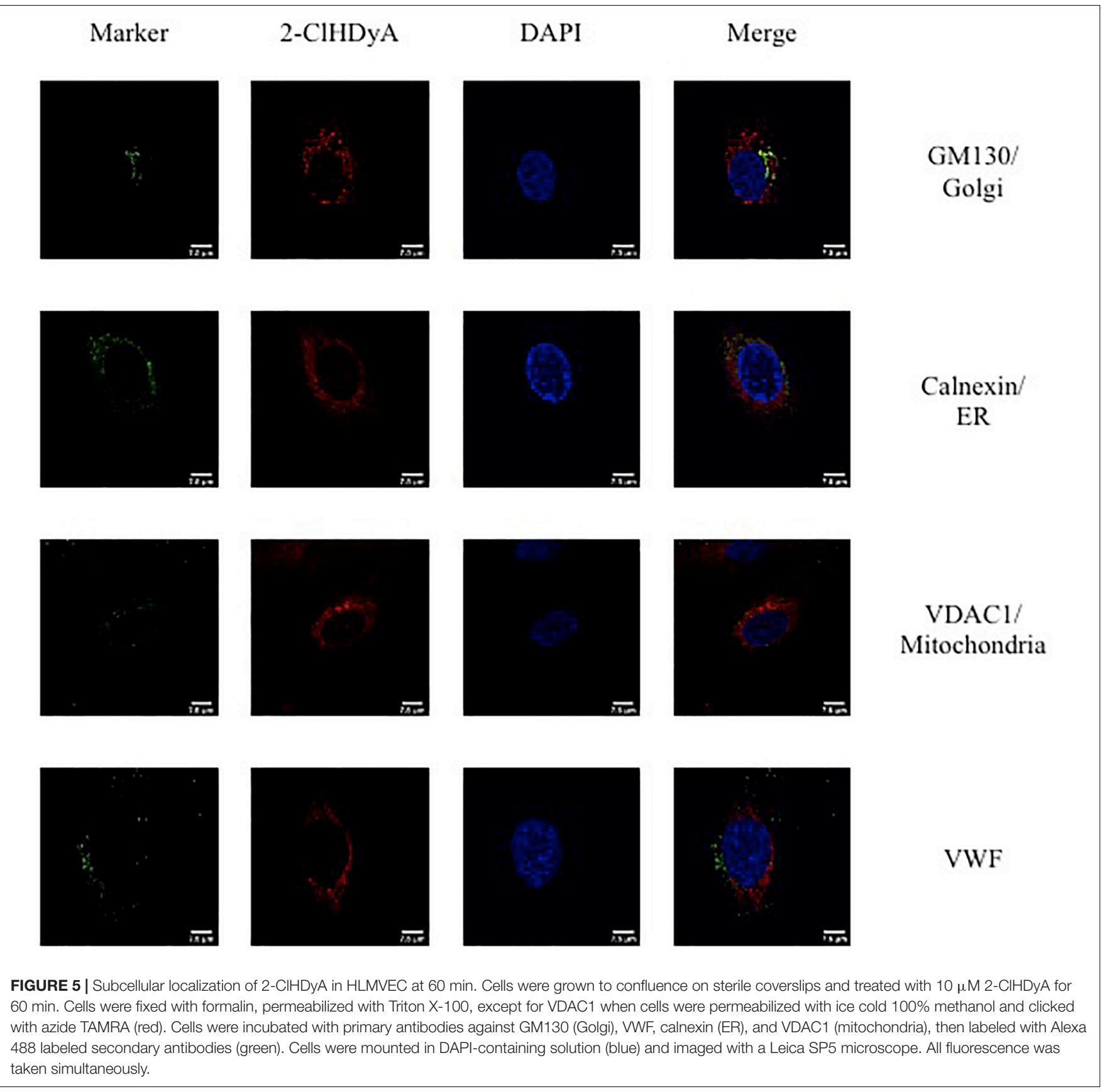

performed using an azide with TAMRA reporter. Colocalization analyses with known organelle markers showed that 2-ClHDyA localized with the ER, Golgi and mitochondria as indicated by calnexin, GM130 and VDAC1 at both 30 and $60 \mathrm{~min}$ in HLMVEC (Figures 4, 5). Additional control experiments showed azide-TAMRA specifically reacts with HLMVEC treated with 2-ClHDyA (Figure 6A, lower panel) and was undetectable in cells that were not treated with 2-ClHDyA (Figure 6A, upper panel). Additional test also showed 2-ClHDyA and 2-ClHDA both elicited barrier dysfunction similar to LPS in HLMVEC (Figure 6B) and did not alter cell metabolic activity as determined by MTT assay (Figure 6C). A similar distribution of 2-ClHDyA with ER, Golgi and mitochondria were observed in HCAEC (Figures 7, 8). 2-ClHDyA did not colocalize with the WeibelPalade bodies (detected with VWF in HCAEC) or VWF storage granules in HLMVEC.

\section{DISCUSSION}

Much of the biological role of chlorinated lipids on endothelial activation have focused on the longer lived chlorinated lipid, 2-ClFA, while the mechanisms underlying biological effects of the 2-ClFA precursor, 2-ClFALD, remain to be explored. Using 


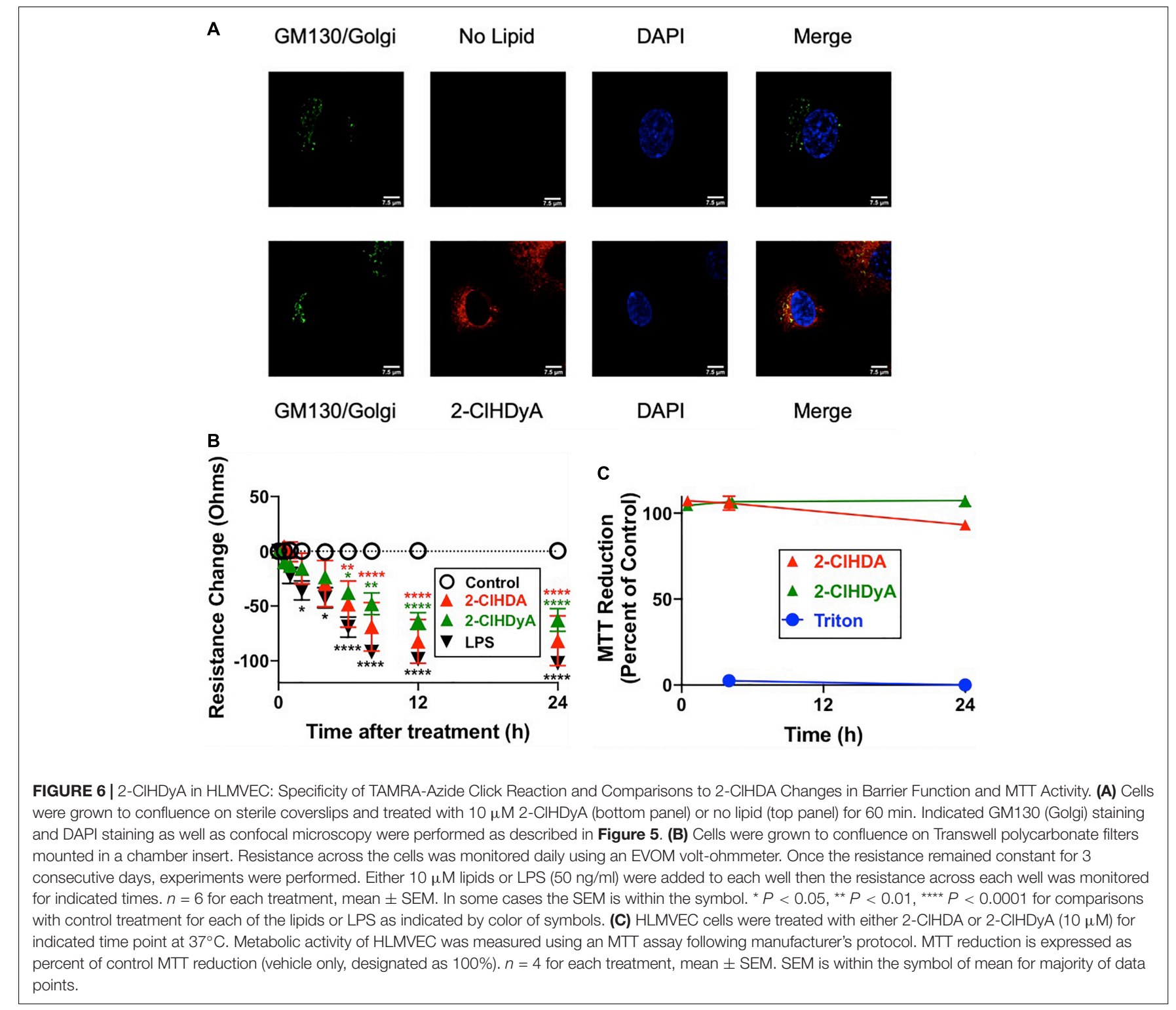

intravital microscopy in the rat we recently showed 2-ClFALD elicits leukocyte rolling and adhesion, as well as permeability changes, in the mesenteric microcirculation. Others have also shown 2-ClFALD causes blood brain barrier dysfunction, but did not examine leukocyte adhesion (Nusshold et al., 2016). These studies also showed 2-CIFALD associated with Golgi, ER and mitochondria in an immortalized human brain endothelial cell line. To enhance our understanding of the biological role of 2ClFALD we now show 2-ClFALD elicits endothelial activation in primary human endothelial cells from three disparate vascular beds (i.e., coronary artery, lung microvascular, and kidney) resulting in adhesion molecule surface expression, neutrophil and platelet adherence, and permeability barrier dysfunction. These findings are significant since they provide important information on tissue beds associated with multi-organ failure during sepsis, and in particular plasma 2-ClFA levels associate with ARDS and 30-day mortality in human sepsis (Meyer et al., 2017).
Human coronary artery endothelial cells were very sensitive to activation by the 2-CIFALD, 2-ClHDA at a concentration $(10 \mu \mathrm{M})$, which is below maximal concentrations estimated at the leukocyte-endothelial interface $(20-90 \mu \mathrm{M})$ (Thukkani et al., 2002; Anbukumar et al., 2010). Robust surface expression of P-selectin, E-selectin, ICAM-1, and VCAM-1 were observed. Neutrophil and platelet adhesion to HCAEC accompanied the surface expression of adhesion molecules in response to 2ClHDA. Interestingly there was robust adhesion of neutrophils and platelets to 2-CIHDA treated HLMVEC and HKEC in the absence of robust increases of surface expression of adhesion molecules. Disparate responses to agonists by endothelial cells originating from different vascular beds have previously been observed (Scott and Patel, 2013), and thus these results most likely represent differences in selectin density and mobilization in response to 2-ClHDA in these cell systems. It can also be speculated that at least for neutrophils, adherence may involve 


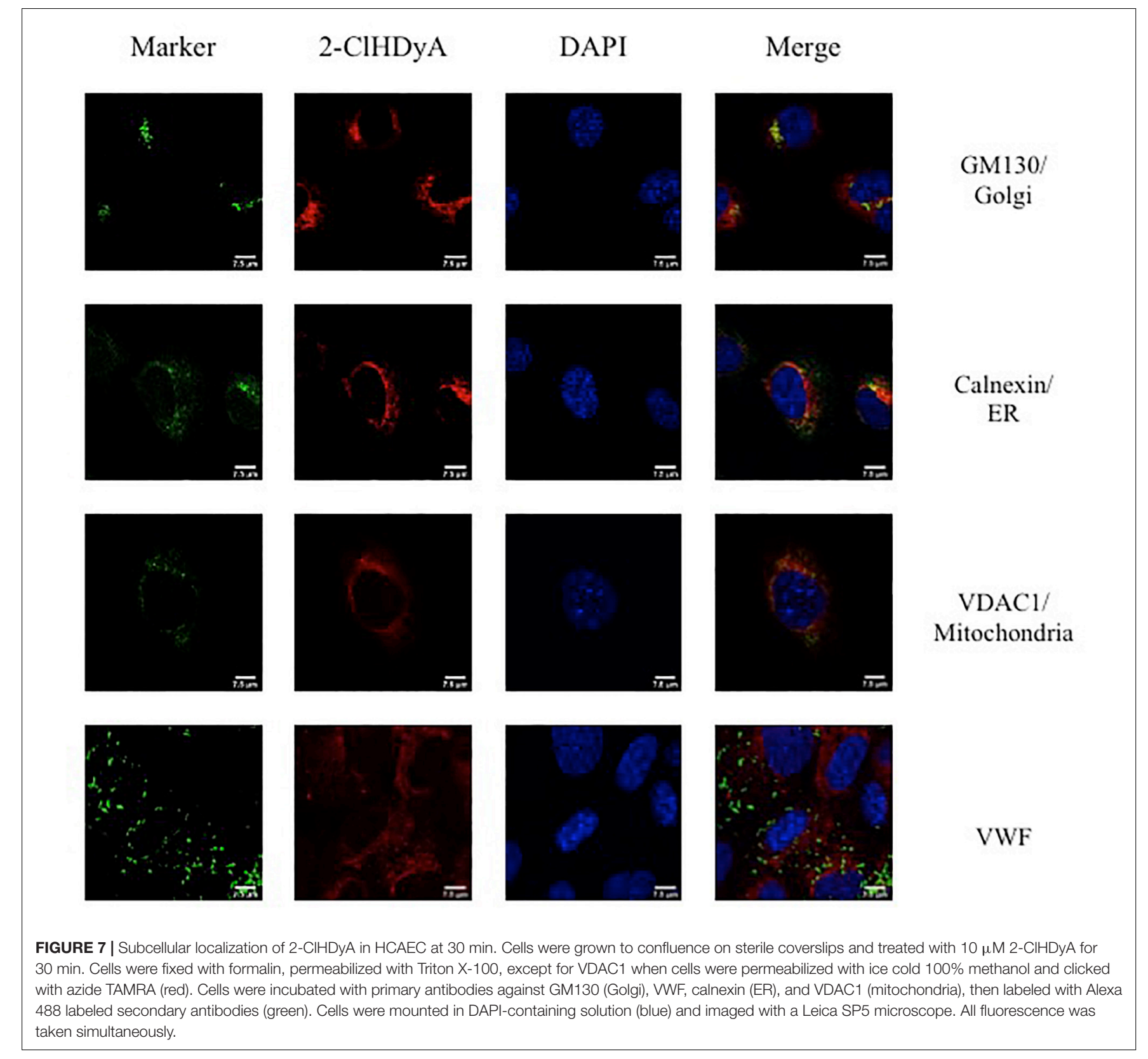

neutrophil netosis, which has been shown to be accelerated by the 2-ClHDA oxidation product, 2-chlorofatty acid (Palladino et al., 2018). Further investigations need to determine if there are novel protein ligands and mechanisms that may be responsible for neutrophils and platelet adherence to 2-ClHDA treated endothelial cells.

We previously used the click-chemistry analog of 2-ClFA to show it selectively associates with the Weibel-Palade bodies of HCAEC and subsequently elicits the cascade of events including P-selectin surface expression and neutrophil adhesion (Hartman et al., 2018). The association of 2-ClFA to the WeibelPalade body suggested the localization may have a key role in mobilizing these storage granules with subsequent surface expression of P-selectin. Similar to 2-ClFA, 2-ClHDA also caused P-selectin surface expression and neutrophil adhesion. However, unlike the click analog of 2-ClFA, 2-ClHDyA did not localize to the HCAEC Weibel-Palade bodies. 2-ClHDyA localized to the ER, Golgi and mitochondria of both HCAEC and HLMVEC. Thus the mechanism responsible for 2-ClHDA mediated mobilization of Weibel-Palade bodies and other granules containing adherence molecules remain to be resolved. In this respect it also should be noted that the HLMVEC contain P-selectin and VWF in separate storage granules (Ochoa et al., 2010; Wu et al., 2014). Future studies need to be performed to determine the mechanism responsible for 2ClHDA as well as 2-ClFA elicited endothelial activation. These studies using confocal microscopy highlight that there may be unique mechanisms responsible for 2-ClHDA and 2-ClFA 


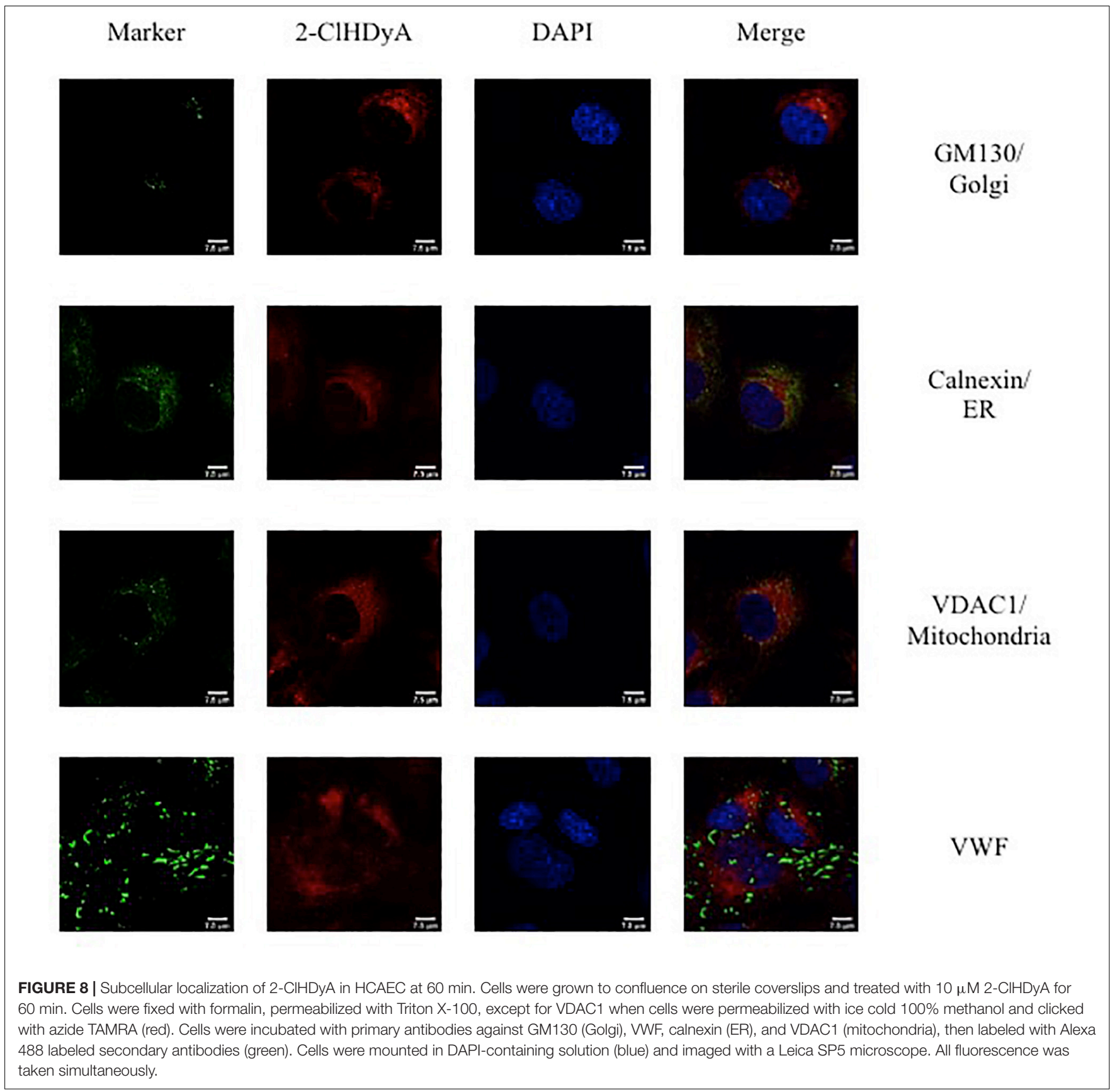

mediated endothelial activation. Additionally the relationship of chlorinated lipids and their association with adhesion molecule receptors needs to be examined.

2-Chlorohexadecanal caused permeability barrier dysfunction in HCAEC, HLMVEC, and HKEC. HDA did have some modest effects in causing endothelial permeability barrier dysfunction, but not to the degree observed with 2-ClHDA treatment. The mechanism responsible for permeability barrier dysfunction may involve adheren dysfunction with intercellular gap formation.

These studies demonstrate that the initial product of $\mathrm{HOCl}$ targeting plasmalogens, 2-ClFALD, activates endothelial cells resulting in adhesion molecule surface expression, neutrophil and platelet adherence and loss of permeability barrier function. There are modest differences in the responses to 2-ClFALD by endothelial cells from three origins including human coronary arteries, human lung, and human kidney. In conjunction with our previous studies using intravital microscopy (Yu et al., 2018), these data suggest this is a key mechanism through which 2CIFALD can elicit in vivo endothelial dysfunction leading to microcirculatory collapse. Several key observations were also made indicating there are additional key gaps that need to be examined in the future including the precise mechanism that 2-ClFALD elicits adhesion molecule surface expression and the mechanisms responsible for platelet and neutrophil adherence to 
2-ClFALD treated endothelial cells in the absence of a response with adhesion molecule surface expression (e.g., in HKEC).

\section{DATA AVAILABILITY STATEMENT}

All datasets generated for this study are included in the article/supplementary material.

\section{ETHICS STATEMENT}

The studies involving human participants were reviewed and approved by the Saint Louis University IRB. The patients/participants provided their written informed consent to participate in this study.

\section{REFERENCES}

Ait-Oufella, H., Maury, E., Lehoux, S., Guidet, B., and Offenstadt, G. (2010). The endothelium: physiological functions and role in microcirculatory failure during severe sepsis. Intensive Care Med. 36, 1286-1298. doi: 10.1007/s00134010-1893-6

Albert, C. J., Crowley, J. R., Hsu, F. F., Thukkani, A. K., and Ford, D. A. (2001). Reactive chlorinating species produced by myeloperoxidase target the vinyl ether bond of plasmalogens: identification of 2-chlorohexadecanal. J. Biol. Chem. 276, 23733-23741. doi: 10.1074/jbc.M101447200

Anbukumar, D. S., Shornick, L. P., Albert, C. J., Steward, M. M., Zoeller, R. A., Neumann, W. L., et al. (2010). Chlorinated lipid species in activated human neutrophils: lipid metabolites of 2-chlorohexadecanal. J. Lipid Res. 51, 10851092. doi: 10.1194/jlr.M003673

Beckett, C. S., Kell, P. J., Creer, M. H., and McHowat, J. (2007). Phospholipase a2-catalyzed hydrolysis of plasmalogen phospholipids in thrombin-stimulated human platelets. Thromb. Res. 120, 259-268. doi: 10.1016/j.thromres.2006. 09.005

Brahmbhatt, V. V., Albert, C. J., Anbukumar, D. S., Cunningham, B. A., Neumann, W. L., and Ford, D. A. (2010). \{omega\}-oxidation of \{alpha\}-chlorinated fatty acids: Identification of \{alpha\}-chlorinated dicarboxylic acids. J. Biol. Chem. 285, 41255-41269. doi: 10.1074/jbc.M110.147157

Chilton, F. H., and Connell, T. R. (1988). 1-ether-linked phosphoglycerides. Major endogenous sources of arachidonate in the human neutrophil. J. Biol. Chem. 263, 5260-5265.

Crea, F., Camici, P. G., and Bairey Merz, C. N. (2014). Coronary microvascular dysfunction: an update. Eur. Heart J. 35, 1101-1111.

De Backer, D., Orbegozo Cortes, D., Donadello, K., and Vincent, J.-L. (2014). Pathophysiology of microcirculatory dysfunction and the pathogenesis of septic shock. Virulence 5, 73-79. doi: 10.4161/viru.26482

Dorman, R. V., Dreyfus, H., Freysz, L., and Horrocks, L. A. (1976). Ether lipid content of phosphoglycerides from the retina and brain of chicken and calf. Biochim. Biophys. Acta 486, 55-59. doi: 10.1016/0005-2760(77) 90069-8

Duerr, M. A., Aurora, R., and Ford, D. A. (2015). Identification of glutathione adducts of alpha-chlorofatty aldehydes produced in activated neutrophils. J. Lipid Res. 56, 1014-1024. doi: 10.1194/jlr.M058636

Etzioni, A. (1996). Adhesion molecules-their role in health and disease. Pediatr. Res. 39, 191-198.

Ford, D. A. (2010). Lipid oxidation by hypochlorous acid: chlorinated lipids in atherosclerosis and myocardial ischemia. Clin. Lipidol. 5, 835-852. doi: 10. $2217 /$ clp. 10.68

Ford, D. A., and Gross, R. W. (1989). Plasmenylethanolamine is the major storage depot for arachidonic acid in rabbit vascular smooth muscle and is rapidly hydrolyzed after angiotensin ii stimulation. Proc. Natl. Acad. Sci. U.S.A. 86, 3479-3483. doi: 10.1073/pnas.86.10.3479

\section{AUTHOR CONTRIBUTIONS}

DF was responsible for oversight of all aspects of studies and manuscript preparation. JM performed endothelial cell functional assays and manuscript preparation. SS performed endothelial cell sub cellular localization studies and manuscript preparation.

\section{FUNDING}

This study was supported (in part) by research funding from the National Institutes of Health R01 GM115553 and R01 GM129508 to DF. The content is solely the responsibility of the authors and does not necessarily represent the official views of the National Institutes of Health.

Ford, D. A., Honavar, J., Albert, C. J., Duerr, M. A., Oh, J.-Y., Doran, S., et al. (2016). Formation of chlorinated lipids post-chlorine gas exposure. J. Lipid Res. 57, 1529-1540. doi: 10.1194/jlr.M069005

Gross, R. W. (1985). Identification of plasmalogen as the major phospholipid constituent of cardiac sarcoplasmic reticulum. Biochemistry 24, 1662-1668. doi: 10.1021/bi00328a014

Halland, N., Braunton, A., Bachmann, S., Marigo, M., and Jorgensen, K. A. (2004). Direct organocatalytic asymmetric alpha-chlorination of aldehydes. J. Am. Chem. Soc. 126, 4790-4791. doi: 10.1021/ja049231m

Harrison, J. E., and Schultz, J. (1976). Studies on the chlorinating activity of myeloperoxidase. J. Biol. Chem. 251, 1371-1374.

Hartman, C. L., Duerr, M. A., Albert, C. J., Neumann, W. L., McHowat, J., and Ford, D. A. (2018). 2-chlorofatty acids induce weibel-palade body mobilization. J. Lipid Res. 59, 113-122. doi: 10.1194/jlr.M080200

Hazen, S. L., Hall, C. R., Ford, D. A., and Gross, R. W. (1993). Isolation of a human myocardial cytosolic phospholipase $\mathrm{a}_{2}$ isoform. Fast atom bombardment mass spectroscopic and reverse-phase high pressure liquid chromatography identification of choline and ethanolamine glycerophospholipid substrates. J. Clin. Invest. 91, 2513-2522. doi: 10.1172/JCI116487

Hazen, S. L., Hsu, F. F., Duffin, K., and Heinecke, J. W. (1996). Molecular chlorine generated by the myeloperoxidase-hydrogen peroxide-chloride system of phagocytes converts low density lipoprotein cholesterol into a family of chlorinated sterols. J. Biol. Chem. 271, 23080-23088. doi: 10.1074/jbc.271.38. 23080

Hsu, F. F., Turk, J., Thukkani, A. K., Messner, M. C., Wildsmith, K. R., and Ford, D. A. (2003). Characterization of alkylacyl, alk-1-enylacyl and lyso subclasses of glycerophosphocholine by tandem quadrupole mass spectrometry with electrospray ionization. J. Mass Spectrom. 38, 752-763. doi: 10.1002/jms.491

Ijzerman, R. G., Jongh, R. T. D., Beijk, M. A. M., Van Weissenbruch, M. M., Delemarre-van De Waal, H. A., Serné, E. H., et al. (2003). Individuals at increased coronary heart disease risk are characterized by an impaired microvascular function in skin. Eur. J. Clin. Invest. 33, 536-542. doi: 10.1046/j. 1365-2362.2003.01179.x

Klebanoff, S. J., Kettle, A. J., Rosen, H., Winterbourn, C. C., and Nauseef, W. M. (2013). Myeloperoxidase: a front-line defender against phagocytosed microorganisms. J. Leukocyte Biol. 93, 185-198. doi: 10.1189/jlb.0712349

Langer, H. F., and Chavakis, T. (2009). Leukocyte-endothelial interactions in inflammation. J. Cell. Mol. Med. 13, 1211-1220.

McHowat, J., Jones, J. H., and Creer, M. H. (1997). Gradient elution reversedphase chromatographic isolation of individual glycerophospholipid molecular species. J. Chromatogr. B Biomed. Sci. Appl. 702, 21-32. doi: 10.1016/s03784347(97)00386- 1

Meyer, N. J., Reilly, J. P., Feng, R., Christie, J. D., Hazen, S. L., Albert, C. J., et al. (2017). Myeloperoxidase-derived 2-chlorofatty acids contribute to human sepsis mortality via acute respiratory distress syndrome. J. Clin. Invest. Insight 2:96432. doi: $10.1172 /$ jci.insight. 96432 
Murphy, E. J., Joseph, L., Stephens, R., and Horrocks, L. A. (1992). Phospholipid composition of cultured human endothelial cells. Lipids 27, 150-153. doi: 10.1007/bf02535816

Nusshold, C., Ullen, A., Kogelnik, N., Bernhart, E., Reicher, H., Plastira, I., et al. (2016). Assessment of electrophile damage in a human brain endothelial cell line utilizing a clickable alkyne analog of 2-chlorohexadecanal. Free Radic. Biol. Med. 90, 59-74. doi: 10.1016/j.freeradbiomed.2015.11.010

Ochoa, C. D., Wu, S., and Stevens, T. (2010). New developments in lung endothelial heterogeneity: von willebrand factor, p-selectin, and the weibel-palade body. Semin. Thromb. Hemost. 36, 301-308. doi: 10.1055/s-0030-1253452

Palladino, E. N. D., Katunga, L. A., Kolar, G. R., and Ford, D. A. (2018). 2chlorofatty acids: lipid mediators of neutrophil extracellular trap formation. J. Lipid Res. 59, 1424-1432. doi: 10.1194/jlr.M084731

Pattison, D. I., and Davies, M. J. (2006). Reactions of myeloperoxidase-derived oxidants with biological substrates: gaining chemical insight into human inflammatory diseases. Curr. Med. Chem. 13, 3271-3290. doi: 10.2174/ 092986706778773095

Pattison, D. I., Hawkins, C. L., and Davies, M. J. (2009). What are the plasma targets of the oxidant hypochlorous acid? A kinetic modeling approach. Chem. Res. Toxicol. 22, 807-817. doi: 10.1021/tx800372d

Peyvandi, F., Garagiola, I., and Baronciani, L. (2011). Role of von willebrand factor in the haemostasis. Blood Transfusion 9, s3-s8. doi: 10.1016/j.thromres.2017. 07.018

Portilla, D., and Creer, M. H. (1995). Plasmalogen phospholipid hydrolysis during hypoxic injury of rabbit proximal tubules. Kidney Int. 47, 1087-1094. doi: 10.1038/ki.1995.155

Rizzo, W. B., Craft, D. A., Dammann, A. L., and Phillips, M. W. (1987). Fatty alcohol metabolism in cultured human fibroblasts. Evidence for a fatty alcohol cycle. J. Biol. Chem. 262, 17412-17419.

Rossaint, J., and Zarbock, A. (2015). Pathogenesis of multiple organ failure in sepsis. Crit. Rev. Immunol. 35, 277-291. doi: 10.1615/critrevimmunol. 2015015461

Santos, S. S., Carmo, A. M., Brunialti, M. K., Machado, F. R., Azevedo, L. C., Assuncao, M., et al. (2016). Modulation of monocytes in septic patients: preserved phagocytic activity, increased ros and no generation, and decreased production of inflammatory cytokines. Intensive Care Med. 4:5. doi: 10.1186/ s40635-016-0078-1

Scott, D. W., and Patel, R. P. (2013). Endothelial heterogeneity and adhesion molecules n-glycosylation: implications in leukocyte trafficking in inflammation. Glycobiology 23, 622-633. doi: 10.1093/glycob/cwt014

Szekanecz, Z., and Koch, A. E. (2004). Vascular endothelium and immune responses: implications for inflammation and angiogenesis. Rheum. Dis. Clin. North Am. 30, 97-114. doi: 10.1016/S0889-857X(03)00116-9

Thukkani, A. K., Albert, C. J., Wildsmith, K. R., Messner, M. C., Martinson, B. D., Hsu, F. F., et al. (2003). Myeloperoxidase-derived reactive chlorinating species from human monocytes target plasmalogens in low density lipoprotein. J. Biol. Chem. 278, 36365-36372. doi: 10.1074/jbc.M305449200

Thukkani, A. K., Hsu, F. F., Crowley, J. R., Wysolmerski, R. B., Albert, C. J., and Ford, D. A. (2002). Reactive chlorinating species produced during neutrophil activation target tissue plasmalogens: production of the chemoattractant, 2-chlorohexadecanal. J. Biol. Chem. 277, 3842-3849. doi: 10.1074/jbc. M109489200

Thukkani, A. K., Martinson, B. D., Albert, C. J., Vogler, G. A., and Ford, D. A. (2005). Neutrophil-mediated accumulation of 2-clhda during myocardial infarction: 2-clhda-mediated myocardial injury. Am. J. Physiol. 288, H2955H2964. doi: 10.1152/ajpheart.00834.2004

Verheul, H. M. W., Jorna, A. S., Hoekman, K., Broxterman, H. J., Gebbink, M. F. B. G., and Pinedo, H. M. (2000). Vascular endothelial growth factorstimulated endothelial cells promote adhesion and activation of platelets. Blood 96, 4216-4221.

Wang, W. Y., Albert, C. J., and Ford, D. A. (2014). Alpha-chlorofatty acid accumulates in activated monocytes and causes apoptosis through reactive oxygen species production and endoplasmic reticulum stress. Arterioscler. Thromb. Vasc. Biol. 34, 526-532. doi: 10.1161/ATVBAHA.113.30 2544

Wildsmith, K. R., Albert, C. J., Anbukumar, D. S., and Ford, D. A. (2006). Metabolism of myeloperoxidase-derived 2-chlorohexadecanal. J. Biol. Chem. 281, 16849-16860.

Wu, S., Zhou, C., King, J. A., and Stevens, T. (2014). A unique pulmonary microvascular endothelial cell niche revealed by weibel-palade bodies and griffonia simplicifolia. Pulmon. Circ. 4, 110-115. doi: 10.1086/67 4879

Ysebaert, D. K., De Greef, K. E., Vercauteren, S. R., Ghielli, M., Verpooten, G. A., Eyskens, E. J., et al. (2000). Identification and kinetics of leukocytes after severe ischaemia/reperfusion renal injury. Nephrol. Dialysis Transpl. 15, 1562-1574. doi: $10.1093 / \mathrm{ndt} / 15.10 .1562$

Yu, H., Wang, M., Wang, D., Kalogeris, T. J., McHowat, J., Ford, D. A., et al. (2018). Chlorinated lipids elicit inflammatory responses in vitro and in vivo. Shock 51, 114-122. doi: 10.1097/SHK.0000000000001112

Conflict of Interest: The authors declare that the research was conducted in the absence of any commercial or financial relationships that could be construed as a potential conflict of interest.

Copyright (c) 2020 McHowat, Shakya and Ford. This is an open-access article distributed under the terms of the Creative Commons Attribution License (CC BY). The use, distribution or reproduction in other forums is permitted, provided the original author(s) and the copyright owner(s) are credited and that the original publication in this journal is cited, in accordance with accepted academic practice. No use, distribution or reproduction is permitted which does not comply with these terms. 